\title{
A MINIMAL COMPACTIFICATION FOR EXTENDING CONTINUOUS FUNCTIONS
}

\author{
PETER A. LOEB ${ }^{1}$
}

Let $W$ be a noncompact, locally compact Hausdorff space, and let $Q$ be a nonvoid set of continuous functions defined on $W$ with each $f \in Q$ having its range in some compact Hausdorff space $X_{f}$. Following the work of Constantinescu and Cornea ([1, pp. 96-97], where $W$ is a Riemann surface and $Q$, a class of extended real-valued functions) one adjoins the continuous real-valued functions with compact support, $C_{0}$, to $Q$ and associates $W$ with its image in the product space $\prod_{f \in Q \cup C_{0}} X_{f}$ under the evaluation map $e$. (For each $x \in W, e(x)(f)$ $=f(x)$.) The closure of $e(W)$ in $\prod_{f \in Q \cup c_{0}} X_{f}$ is a compact Hausdorff space $\bar{W}$ containing $W$ (i.e. $e(W)$ ) as a dense subset, and the functions in $Q$ have continuous extensions to $\bar{W}$ which separate the points of $\bar{W}-W$. Any compactification of $W$ with these properties is homeomorphic to this one and is called a $Q$-compactification of $W$.

Below, we construct a $Q$-compactification of $W$ which uses only the product of the spaces $X_{f}$ for $f$ in $Q$. Thus we avoid using the axiom of choice if $Q$ is a countable collection of real- or complex-valued functions (see [3]), and for many examples we obtain a compactification which is easier to visualize than the one given above.

TheOREM. Let $Y$ be the product space $\prod_{f \in Q} X_{f}$ and $e$ the evaluation map sending $W$ into $Y$. Set $\Delta=\cap\left\{[e(W-K)]^{-}: K\right.$ compact, $\left.K \subset W\right\}$, and let $\tilde{W}=W \cup \Delta$. Given a point $x$ in $\Delta$, an open set $N$ in the standard base for the topology of $Y$ with $x \in N$, and a compact set $K \subset W$, we set $N(x, K)=[N \cap \Delta] \cup\left[e^{-1}(N)-K\right]$. If 3 is the topology on $\tilde{W}$ generated by the base consisting of all open sets in $W$ and all the sets $N(x, K)$, then $(\tilde{W}, J)$ is a $Q$-compactification of $W$.

The proof of the theorem is not hard; the compactness of $\tilde{W}$ follows from the fact that a net which is eventually in the complement of every compact subset of $W$ has a cluster point in $\Delta$. (See $[2$, p. 136].) Note that if $Q$ is the restriction of the continuous realvalued functions with period 1 to the interval $W=\{x: 0<x \leqq 1\}$, then $e(W)$, which is compact, is not homeomorphic to $W$ even though $Q$ separates the points of $W$. Moreover, $\Delta$ consists of the single point $e(1)$, which is distinct from 1 in $\tilde{W}$. If $W=\{x: 0<x \leqq 1\}$ and

Received by the editors April 6, 1966.

${ }^{1}$ Supported by National Science Foundation research grant GP-5279. 
$Q=\{\sin (\pi / x)\}$, then $\Delta=\{y:-1 \leqq y \leqq 1\}$ and a typical basic neighborhood of a point $y_{0} \in \Delta$ is given by positive constants $\delta$ and $\epsilon$ and has the form

$\left\{y \in \Delta:\left|y-y_{0}\right|<\epsilon\right\} \cup\left\{x \in W: x<\delta\right.$ and $\left.\left|\sin (\pi / x)-y_{0}\right|<\epsilon\right\}$.

REFERENCES

1. C. Constantinescu and A. Cornea, Ideale ränder Riemannscher Flächen, Ergebnisse der Math., Vol. 32, Springer, Berlin, 1963.

2. J. L. Kelley, General topology, Van Nostrand, Princeton, N. J., 1955.

3. P. A. Loeb, $A$ new proof of the Tychonoff theorem, Amer. Math. Monthly 72 (1965), 711-717.

University of California, Los Angeles 